\title{
In eigener Sache
}

\section{Sabine Kropp}

Seit dem DVPW-Kongress vom vergangenen September 2010 - dem im Heft 4/2009 ein ausführlicher Bericht über die Tätigkeit der Redaktion folgte - haben sich in der PVS zwar keine inhaltlichen, aber doch einige personelle Veränderungen ergeben. Ruth Zimmerling, die in der Redaktion für den Bereich „Politische Theorie“ sowie für den Literaturteil der Zeitschrift verantwortlich zeichnete, hat diese Aufgabe zum 1. März 2010 nach mehr als fünf Jahren abgegeben. Wir danken Ruth Zimmerling für ihren überaus engagierten, langjährigen Einsatz sehr herzlich. Es war uns stets eine große Freude, mit einer so zuverlässigen und kompetenten Kollegin, auf deren Rat man stets bauen konnte, zusammenzuarbeiten, und wir wünschen ihr für ihre künftigen Pläne und Vorhaben alles erdenkliche Gute und viel Erfolg. Da sich der Arbeitsumfang für die Betreuung eines Teilbereichs unseres Faches und des Literatur- und Rezensionsteils der PVS als doch sehr aufwendig erwiesen hat, hat sich die Redaktion im Benehmen mit Vorstand und Beirat der DVPW dazu entschlossen, diese beiden Bereiche künftig personell zu trennen. Rainer Schmalz-Bruns hat inzwischen als Redakteur die Verantwortung für den Bereich „Theorie“ übernommen; Emanuel Richter betreut ebenfalls seit März 2010 den Literaturteil der Zeitschrift. Beide Kollegen heiße ich herzlich - auch im Namen meiner Kolleginnen und Kollegen - im Redaktionsteam willkommen: Wir freuen uns auf eine enge und vertrauensvolle Zusammenarbeit.

Selbiges gilt auch für Gabriele Abels, die sich seit März 2010 als Redakteurin insbesondere um den Bereich „Europa und Policy-Analyse“ kümmert: auch ihr ein herzliches Willkommen! Mit der Wahl einer weiteren Kollegin in das Redaktionsteam tragen Herausgeber und Redaktion nicht zuletzt der zunehmenden Ausdifferenzierung unseres Faches Rechnung. Die Arbeit kann durch die Aufstockung von vier auf nunmehr sechs Redakteure zudem auf mehr Schultern verteilt werden. Gleichwohl bleibt es auch weiterhin bei der inzwischen eingeübten und bewährten Praxis des Gegenlesens eines jeden Manuskripts durch die anderen Redakteure, die eine breite Meinungsbildung erst ermöglicht.

\footnotetext{
Online publiziert: 26.05 .2010

(C) VS-Verlag 2010

Univ. Prof. Dr. S. Kropp $(\bowtie)$

Lehrstuhl für Politikwissenschaft,

Deutsche Hochschule für Verwaltungswissenschaften Speyer,

Freiherr-vom-Stein-Straße 2,

67346 Speyer, Deutschland

E-Mail: pvs@dhv-speyer.de
} 
Schließlich freue ich mich, dass wir mit Marie-Sophie Heinelt eine Redaktionsassistentin gewinnen konnten, die den alltäglichen Redaktionsbetrieb nach dem Ausscheiden von Priska Schorlemmer schnell, zuverlässig und engagiert übernommen hat. Manuskripte können somit künftig unter heinelt@dhv-speyer.de bei der PVS-Redaktion eingereicht werden. Wir danken bei dieser Gelegenheit auch Frau Schorlemmer für ihren Einsatz und die mehrjährige gute Zusammenarbeit und wünschen ihr für ihren weiteren Werdegang alles Gute und viel Erfolg. 